\title{
Emprendimiento transgeneracional en la empresa familiar: factor clave para la competitividad de las MIPYMES
}

\section{Transgenerational entrepreneurship in the family business: key factor for the competitiveness of MIPYMES}

\author{
María José Pérez Espinoza \\ Universidad Metropolitana del Ecuador, Ecuador \\ Autor para correspondencia: mjperez@umet.edu.ec \\ Fecha de recepción: 20 de julio de 2018 - Fecha de aceptación: 30 de agosto de 2018
}

Resumen: Perdurar con éxito en el tiempo es uno de los principales objetivos de toda empresa, siendo aún éste más importante en las empresas familiares. La longevidad depende en gran parte de la capacidad de emprender de la familia empresaria, generación tras generación. Esta capacidad permite que la empresa familiar desarrolle valores, y genere recursos y capacidades en los miembros de la familia que permitan desarrollar nuevas ideas de negocio, nuevos productos que creen valor social y financiero llegando a tener una ventaja competitiva sostenible en el tiempo. La competitividad es un término que se encuentra estrechamente relacionado con las empresas indiferentemente de su tamaño, sin embargo en el caso de las MIPYMES resulta transcendental tenerlo presente considerando el hecho de que de ello dependerá su permanencia en el mercado. Desde luego, la principal fuente de creación de empresas es mérito de los emprendedores que día a día se arriesgan a sumergirse en la aventura de empezar una empresa a partir de pequeñas necesidades identificadas, esto sobre todo se da con bastante frecuencia en las empresas familiares, las mismas que de acuerdo a fuentes estadísticas representan más del $80 \%$ de las empresas que forman parte del sector económico. En la presente investigación se quiere presentar un análisis completo y objetivo acerca del vínculo existente entre la competitividad, el emprendimiento y su presencia e influencia en las empresas sobre todo de tipo familiar que llegan a ser transgeneracionales justamente por la identificación a tiempo de nuevos nichos de mercado, líneas de negocio, o innovaciones a la tradicional empresa de familia.

Palabras Claves: empresas familiares; emprendimiento transgeneracional; competitividad; MIPYMES

Abstract: To last with success in the time, is one of the main objectives of all enterprise, and even more important in the family companies. The longevity depends mainly in the entrepreneur capacity of the business family, generation after generation. This capacity allows that the family business develops values, and generates resources and capacities in the family members that let them to develop new business ideas, new products that create social and financial value and in that way to reach a competitive advantage in the time. The competitiveness is a term that is closely related with the enterprises indifferently of their sizes. Certainly, the principal source of business creation is responsibility of the entrepreneurs that daily take the risk to immerse in the adventure to start a company taking into account the small identified needs, mainly in family businesses, that according to statistical sources represent more than $80 \%$ of the companies that are part of the 
economic sector. The objective of this research is to present a complete and objective analysis about the relationship between the competitiveness, the entrepreneurship and its presence and influence in the companies mainly in those who are considered as familiar that reach to be transgenerational justly for the timely identification of new market niches, business lines or innovations to the traditional family enterprise.

Key Words: family business; transgenerational entrepreneurship; competitiveness; PYMES

\section{Introducción}

En el Ecuador, no se puede mencionar el sector económico empresarial sin tomar en cuenta que este se encuentra conformado principalmente por pequeñas y medianas empresas de tipo familiar, siendo estadísticamente más exactos alrededor del 80\% de los negocios cumplen con ambas características (Ecuador en Cifras, 2018), dicho porcentaje permite denotar que desde hace varios años atrás ha existido una fuerte tendencia de creación o crecimiento de negocios que no hace variar la cifra a la baja. Pero ¿a qué se debe la permanencia de este indicador?, pues bien, muchos expertos lo atribuyen a la tendencia a emprender de los considerados millennials, que quieren ser dueños de su tiempo, su dinero y empoderarse completamente de las actividades que realizan (Ortega, 2014), características que desde luego se encuentran presentes en las nuevas generaciones que empezaran a tomar el mando en las empresas familiares de la economía ecuatoriana. Las MIPYMES, son pequeñas células económicas que alimentan el día a día de la actividad mercantil del país, por tal razón constituyen un factor clave dentro del desarrollo socioeconómico de cualquier ciudad o entorno en el que se encuentren, ya que no solamente se enfocan en mejorar las operaciones comerciales de la actividad a la cual se dedican, sino que también reducen el desempleo, mejoran la calidad de vida de las personas que trabajan en ellas y dinamizan la economía en su conjunto (Yance, Solis, Burgos, \& Hermida, 2017). Pero así como las MIPYMES realizan grandes aportes a la economía local, así también se enfrentan a grandes retos dependiendo de la época en la cual se encuentren, uno de los retos del siglo XXI de acuerdo a Álvarez (2016) de la mano de la competitividad y el desarrollo sostenible es el de crear bienes y servicios para los clientes potenciales pero que tengan un mayor nivel de tecnología, por lo cual las empresas ecuatorianas han empezado a alinear todas sus estrategias para competir más agresivamente en el mercado local y de esta manera comenzar a considerar la competencia fuera de los limites nacionales y llegar a otros niveles de producción y distribución a nivel de región o continente.

Tomando como foco principal a las MIPYMES de tipo familiar se puede identificar que una de sus ventajas competitivas es que los propietarios o fundadores crecen en la misma medida en que lo hace el negocio, de tal manera que con el pasar de los años logran un nivel de especialización asombrosa con respecto a otros competidores no familiares (Miller \& Le BretonMiller, 2015), sin embargo la especialización que puede alcanzar la compañía no es garantía de su permanencia a largo plazo en el mercado, y es ahí cuando el emprendimiento transgeneracional empieza a tomar partido dentro de dicha ecuación, ya que permite desarrollar nuevas oportunidades en mercados emergentes o nuevas líneas de negocio que apunten a nichos de mercado diferentes a los existentes en la actualidad.

\section{La competitividad de las Mipymes}


De acuerdo al Índice de Competitividad Global del periodo 2016-2017 el Ecuador se ubicó en el puesto 91 de 136 países con una calificación de 3.94, un considerable descenso frente al periodo pasado que se encontraba en el puesto 76 (Foro Económico Mundial , 2016), este dato resulta de suma importancia para el entorno empresarial general del país, ya que repercute directamente en la manera de negociar con respecto a otros países alrededor del mundo, ¿será acaso que este índice puede repercutir en la permanencia de las mipymes en el mercado?, pues la respuesta es que sí, cada indicador económico sea este micro o macro afectará al desarrollo de la economía en su conjunto y a todas las empresas que la conforman, sean estas grandes, pequeñas, medianas o micro.

Los tres factores principales que aniquilan la competitividad empresarial del país son: las medidas laborales restrictivas, la dificultad para acceder a financiamiento y los impuestos que se aplican tanto para comprar como para vender o comercializar mercadería dentro y fuera del país (Foro Económico Mundial , 2016), factores que dependen en su totalidad de las políticas económicas del gobierno de turno del país, sin embargo las empresas deben poner a volar su creatividad estratégica para poder tomar medidas que de alguna manera las ponga en el radar de clientes potenciales que quieran incursionar en nuevos mercados y probar bienes o servicios diferentes.

A pesar de que el término "competitividad" se encuentra con mucha frecuencia en todo lo relacionado al sector empresarial, este puede ser aplicado en diferentes campos y desde diferentes perspectivas, por tal razón resulta importante hacer hincapié en el punto de vista que se lo está analizando en la presente investigación, con dicho antecedente se puede tomar de referencia la definición desarrollada por Mora-Riapira, Vera-Colina y Melgarejo-Molina (2015) que menciona que la competitividad empresarial está asociada a lo que ocurre fuera de la organización (políticas gubernamentales, estructura económica del país, características del mercado, variables regionales), pero depende estrechamente del desempeño interno de la misma entidad (p.80), la cual en un contexto netamente empresarial de MIPYMES toma un alto grado de relevancia, ya que denota un equilibrio tanto en los factores internos como externos que pueden afectar a la empresa (Aragón, Rubio, Serna, \& Chabié, 2010).

En la medida en que el mundo y el entorno vayan cambiando con mayor agresividad, en la misma medida las empresas se enfrentarán a panoramas cada vez más inestables y cambiantes, y como se mencionó anteriormente ese vínculo tan fuerte de la competitividad empresarial con factores externos ajenos al manejo propio de la organización hacen que esta deba tomar en consideración ciertos factores considerados como claves tanto para MIPYMES de tipo familiar como no familiar, los cuales son: 1) manejo exitoso de flujos de producción, materia prima e inventarios, 2) manejo exitoso de la interacción de mecanismos entre la planificación, el marketing, investigación y desarrollo, diseño e ingeniería relacionados a la producción industrial (aplicable tanto para bienes como para servicios), 3) la capacidad de combinar las actividades internas de innovación así como de investigación y desarrollo con la cooperación tecnológica tanto con universidades como con otras empresas, 4) la capacidad de organizar exitosamente relaciones de emprendimiento con proveedores de materiales y componentes y con clientes, 5) los pasos para mejorar las capacidades de los obreros y empleados a través de la inversión en entrenamiento especializado, y la generación de niveles más altos de responsabilidad en la producción (Solleiro \& Castañón, 2005). La consideración de estos factores permitirá afianzar el 
concepto de competitividad en general en las MIPYMES que los apliquen y los tengan claro en su radar de planificación.

\section{El emprendimiento como clave del desarrollo empresarial}

Frente a los inminentes cambios del entorno en el cual se desarrollan los negocios, indiferentemente de su tamaño, el emprendimiento ha llegado a ser considerada desde hace varios años atrás la clave empresarial camino hacia el éxito (Valencia-Arias, Gutiérrez, \& Montoya, 2017), ¿a qué se debe dicha consideración?, pues sobre todo la globalización ha modificado competitivamente los entornos del mundo empresarial y por ende han variado transcendentalmente las necesidades y características sociales, culturales, económicas, políticas, etc, de los consumidores potenciales (Buil \& Rocafort, 2016).

Valencia-Arias, Gutiérrez y Montoya (2017) consideran que de acuerdo al sin fin de evidencias encontradas, es cada vez más fuerte el lazo que une al emprendimiento con la competitividad, ya que eso ha dado origen a lo que actualmente se conoce como emprendimiento estratégico, definido por los mismos autores como aquellas actividades que se realizan para explotar las ventajas competitivas que tienen en la actualidad (Leach, 2018), a la par que también realizan una exploración de las innovaciones, investigación y desarrollo de nuevos productos o servicios que serán la base para las futuras ventajas competitivas de la empresa.

Con el planteamiento realizado, puede surgir otra interrogante, ¿será acaso posible adaptar el emprendimiento estratégico a MIPYMES de tipo familiar?, pues desde luego la respuesta es afirmativa, ya que representa un detalle de composición estructural de la organización, mas no un hecho que genere cambios en áreas claves como las de producción, distribución, almacenamiento o administración en general (Padilla-Martínez, Quispe-Otacoma, Noqueira-Rivera, \& Hernández-Nariño, 2016). El emprendimiento y su evolución (por asignarle una denominación particular) conocido como emprendimiento estratégico, permite analizar de una manera mucho más metódica la manera en la cual se empezaran a implementar los nuevos negocios que se desarrollen, ya sean estos establecidos por primera vez en una nueva empresa o en una compañía ya existente a través de la figura de nuevas líneas de negocio (Minniti, 2012).

El emprendimiento se ha caracterizado por tener presente dos elementos primordiales dentro de su definición: estos son la creatividad y la innovación (Valencia-Arias, Gutiérrez, \& Montoya, 2017), sin embargo resulta diferente su aplicación en el caso de un negocio completamente nuevo a un negocio ya previamente establecido que busca innovar su oferta en el mercado, en el primer caso este se caracteriza por: el alto grado de riesgo del negocio como tal, la posibilidad de crecimiento o declive total y desde luego la presentación de una propuesta completamente innovadora, cabe destacar que en este caso el respaldo o prestigio de la marca es nulo, ya que es un negocio completamente nuevo que no garantiza absolutamente nada a sus propietarios o inversionistas (Sánchez \& Pérez, 2015).

En lo que respecta al emprendimiento empresarial ${ }^{1}$ Leach (2018) afirma que en la actualidad es parte de una estrategia de supervivencia, ya que lleva a las empresas a cambiar e

\footnotetext{
${ }^{1}$ Término utilizado para hacer referencial al emprendimiento dentro de una empresa ya creada (Valencia-Arias, Gutiérrez, \& Montoya, 2017)
} 
innovar obligatoriamente con el fin de responder a crisis, cambios, ingreso de nuevos competidores y un sinfín de razones, en otras palabras lleva a las empresas y a sus propietarios o directivos a renovarse para cambiar la dirección estratégica del negocio, la forma en que han venido haciendo las cosas, lo cual desencadena a mediano o largo plazo una mejora del posicionamiento competitivo de la marca, del producto, del servicio y en general de todos aquellos atributos que son altamente valorados por los clientes (Peltola, 2012)

\section{El proceso de sucesión y emprendimiento de generación en generación}

Una vez que se han clarificado y contextualizado dos conceptos claves dentro de la presente investigación, como lo es la competitividad y el emprendimiento, estos serán aterrizados al entorno de las empresas familiares, para lo cual resulta importante conocer a mayor profundidad acerca del proceso de sucesión y la manera en que el emprendimiento puede influir en la permanencia de las empresas en el mercado.

La sucesión de acuerdo a Tápies (2018) es el relevo generacional que protagoniza la siguiente generación en el proceso de sustitución de la generación que la antecede (p.11), es decir aquel paso al costado del fundador o de los "mayores" de la organización, para que las nuevas generaciones tomen la dirección y mando de la compañía. Este proceso se da tanto en empresas de tipo familiar como no, sin embargo, las primeras son estudiadas a mayor profundidad dado el nivel emocional familiar que se encuentra inmiscuido en el cambio generacional y que si no se maneja de una manera adecuada puede llegar a entorpecer todo el proceso sucesorio (Auletta, Monteferrante, \& Rodríguez, 2013).

Varios expertos a lo largo de los años han tratado de crear métodos, estrategias, procesos, metodologías, en fin, un sin número de elementos que sirvan de herramienta para los directivos de las empresas familiares y de esta manera tratar de disminuir el impacto que causa el proceso sucesorio y garantizar de esta manera una mayor permanencia en el mercado cambiante actual (Burgoa, Herrera, \& Treviño, 2013), sin embargo una de las estrategias más acertadas ha sido la empíricamente aplicada por varias empresas conocido como el emprendimiento transgeneracional, que básicamente se resumiría al emprendimiento que se da de generación en generación.

El emprendimiento transgeneracional de acuerdo a D’Aveni (2015) es el resultado que obtienen aquellas familias que en su visión de trascendencia son capaces de generar nuevas actividades económicas a través de las generaciones, creando valor y riqueza sostenida en el tiempo, construyendo así un legado familiar empresarial (p.1), con lo cual surge un nuevo cuestionamiento acerca de este tipo de emprendimiento ¿en qué momento se lo debe realizar para que genere buenos resultados?, pues no existe una repuesta adecuada a dicha pregunta, ya que dependerá en gran medida de la cultura de empresa, de la familia empresaria y en sí del mercado y del tipo de negocio en el cual se encuentre la compañía, y desde luego si los propietarios (familia empresaria) está dispuesta a asumir el riesgo que conlleva tener al emprendimiento dentro del radar de planificación estratégica (Ogliastri, 2013). A continuación se muestra la gráfica del proceso emprendedor de acuerdo a la GEM, en la cual se toman en consideración los tiempos, las características particulares de la actividad emprendedora y aquellos imprevistos que se pueden suscitar entorno a ella. 


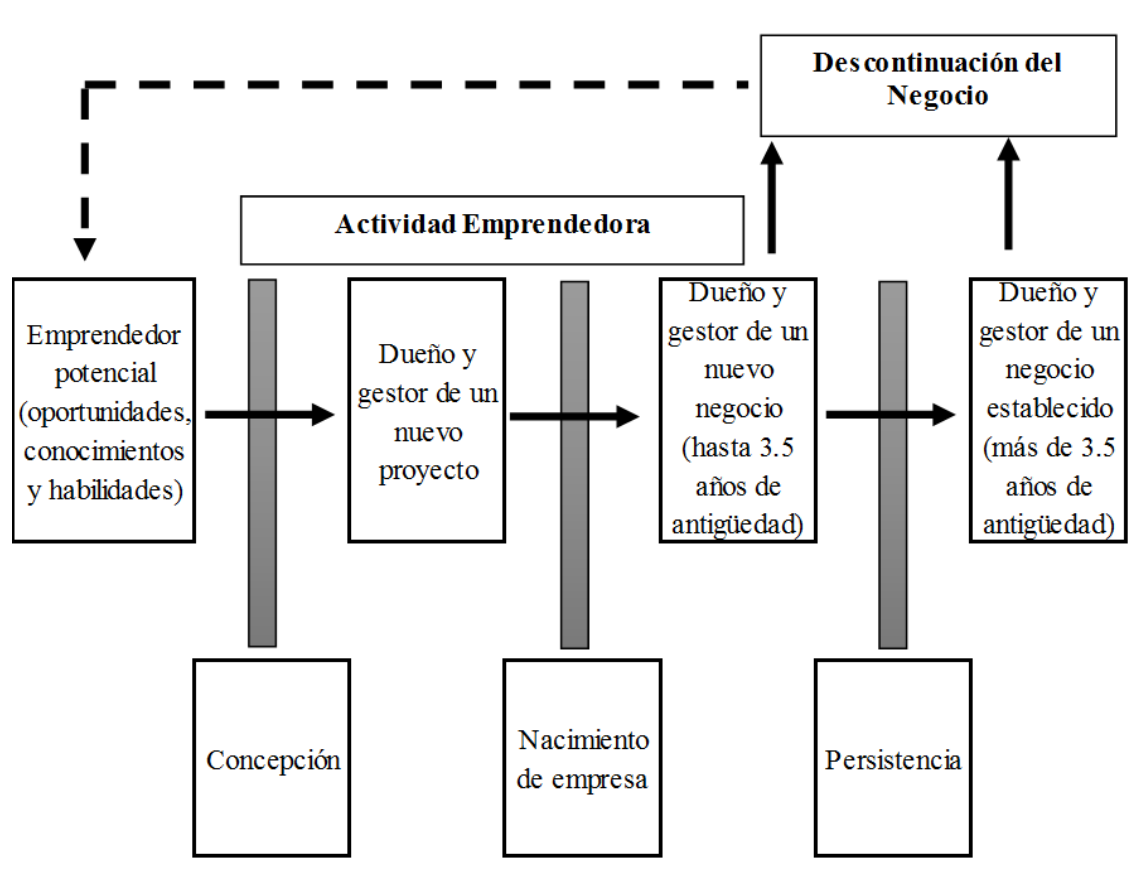

Gráfico 1: El proceso emprendedor de GEM (Global Entrepreneurship Monitor) Fuente: (Landsdale, Abad, \& Vera, 2012)

Dentro del emprendimiento transgeneracional, se debe analizar con minucioso cuidado al principal participante de esta ecuación, es decir la familia emprendedora, la cual más que estar enfocada en el proceso de sucesión en sí, se enfoca en la permanencia de la compañía en el mercado a través del lanzamiento de productos (bienes o servicios) que creen valor, y que se encuentren tomando una oportunidad latente en el mercado (D’Aveni, 2015). Las familias empresarias que tienen como norte la visión de emprender de generación en generación entienden que una de las tareas dentro de su rol en la compañía a más de administrar o gerenciar, es de generar nuevas actividades que inyecten dinero antes de que las utilidades empiecen a mermar, de esta manera se garantiza una permanente fuente de circulante que le dará a la compañía la opción de invertir en nuevos proyectos, mercados o procesos productivos (Rodríguez-Zapatero, Rodríguez-Jiménez, \& Rodríguez-Alcaide, 2015).

Para que el emprendimiento transgeneracional fluya dentro de la cultura empresarial de una organización Ojeda y Chu de Fung (2013) afirman que debe existir una correlacion positiva entre la hostilidad que puede estar presente en el entorno y la orientación emprendedora de la empresa, ya que en los ambientes más dificiles es cuando las empresas se encuentran con mayor apertura de hacer cosas diferentes, por tal razón se debe estar atento a aquellos factores ambientales que motivan sobre todo a las familias a desarrollar nuevos mercados, estas pueden ser inseguridad jurídica, barreras administrativas, amenazas contra la propiedad privada, constantes cambios en regulaciones, entre otros, sin embargo con un excelente espiritu emprendedor inculcado desde el fundador, excelentes relaciones tanto internas como externas y una visión de innovar actividades, se puede ir por el camino correcto del emprendimiento transgeneracional. 


\section{Conclusiones}

En la actualidad las empresas, sobre todo las consideradas como MIPYMES, representan para el mercado un gran aporte económico, ya que no solamente impulsan a la dinamización de la economía, sino que también generan fuentes de empleo que mejoran la calidad de vida de la población en general. En América Latina, existe un gran auge de emprendimiento focalizado en la creación de MIPYMES, sin embargo con el paso del tiempo estas fracasan o no trascienden como se espera, y es que básicamente de eso se trata el emprendimiento, de fracasar, asumir riesgos, borrar lo que se ha hecho y volver a empezar, pero dicho proceso requiere de tiempo y de un capital de inversión amplio para retomar continuamente las actividades.

El caso de las empresas familiares no es la excepción, por tal razón, los fundadores de este tipo de empresas tienen una gran responsabilidad frente a las generaciones venideras, la cual radica en mantener siempre como un objetivo estratégico la innovación constante de las actividades que realizan, ya sea dentro del bien o servicio que ofrecen o a través de la creación de nuevas líneas de negocio en la empresa, por tanto el emprendimiento transgeneracional dependerá en gran medida de la cultura familiar y del espíritu incansable de la familia por tomar nuevos retos y generar valor para ofertar productos diferentes en un mercado competitivo.

\section{Agradecimiento}

Se ofrece un sincero agradecimiento a la Universidad Metropolitana, por brindar el apoyo a la investigación a través del proyecto "Propuestas de mejora de la competitividad empresarial de la MIPYME de la provincia de El Oro", este artículo emerge como un resultado parcial dentro del conjunto de actividades del proyecto de investigación.

\section{Bibliografía}

Álvarez, A. M. (2016). Retos de América Latina: Agenda para el desarrollo sostenible y negociaciones del siglo XXI. Revista Problemas del Desarrollo, 186(47), 9-30.

Aragón, A., Rubio, A., Serna, A., \& Chabié, J. (2010). Estrategia y competitividad empresarial: Un estuido en las MIPYMES de Tabasco. Investigación y Ciencia(47), 4-12.

Auletta, N., Monteferrante, P., \& Rodríguez, A. (2013). Resiliencia ante todo: ¿Cómo superan las crisis las empresas familiares? Debates IESA, 18(1), 23- 27.

Buil, M., \& Rocafort, A. (2016). Emprendimiento y supervivencia empresarial en época de crisis: El caso de Barcelona. Intangible Capital, 12(1), 95-120.

Burgoa, T., Herrera, E., \& Treviño, J. (2013). Estudio sobre la Administración de Empresas Familiares en México: Principales Problemas y Retos que Enfrentan. Daena: International Journal of Good Conscience, 8(2), 1-22.

D’Aveni, R. (2015). Emprendimiento Transgeneracional y Creación de riqueza. Proteus Management Consulting(4), 1-5. 
Ecuador en Cifras. (13 de Julio de 2018). INEC . Obtenido de Instituto Nacional de Estadísticas y Censos : $\quad$ http://www.ecuadorencifras.gob.ec/documentos/webinec/Bibliotecas/Libros/Panorama\%20Laboral\%202017.pdf

Foro Económico Mundial . (2016). Reporte de Competitividad Global 2016-2017. Geneva: WEF. Landsdale, D., Abad, C., \& Vera, D. (2012). Impulsores claves para establecer el ecosistema dinámico de emprendimiento en Ecuador. Revista de la Universidad San Francisco de Quito Polemika, 9(1), 18-25.

Leach, R. (2018). Entrepreneurs, small business owners know nobody grows alone . Crain's Cleveland Business, 9.

Miller, D., \& Le Breton-Miller, I. (2015). Pequeñas grandes lecciones de las empresas familiares. IESE Insight, 33-40.

Minniti, M. (2012). El emprendimiento y el crecimiento económico de las naciones. Economía Industrial(383), 23-30.

Mora-Riapira, E., Vera-Colina, M., \& Melgarejo-Molina, Z. (2015). Planificación estratégica y niveles de competitividad de las Mipymes del sector comercio en Bogotá. Estudios Gerenciales, 31, 79-87.

Ogliastri, E. (2013). ¿Empresa familiar emprendedora? Debates IESA, 18(1), 13-18.

Ojeda, E., \& Chu de Fung, S. (2013). Entender y fomentar el emprendimiento en empresas familiares. Debates IESA, 18(1), 32-36.

Ortega, I. (2014). Millennials: Inventa tu empleo. España : Universidad Internacional de La Rioja (UNIR Editorial).

Padilla-Martínez, M., Quispe-Otacoma, A., Noqueira-Rivera, D., \& Hernández-Nariño, A. (2016). Diagnóstico y perspectivas de fomento del emprendimiento como instrumento de desarrollo. Ingeniería Industrial, 37(1), 91-103.

Peltola, S. (2012). Can an old firm learn new tricks? A corporate entrepreneurship approach to organizational renewal. Business Horizons, 43 53 doi:https://doi.org/10.1016/j.bushor.2011.09.002

Rodríguez-Zapatero, M., Rodríguez-Jiménez, M., \& Rodríguez-Alcaide, J. (2015). La familia empresaria: Capacidad Emprendedora transgeneracional . Cuaderno de Investigación de la Cátedra PRASA de empresa familiar, 3-13.

Sánchez, L., \& Pérez, E. (2015). Las entidades de economía social como protagonistas de un nuevo modelo de emprendimiento y medidas legales de apoyo al emprendimiento. Revista de Economía Pública, Social y Cooperativa(84), 35-62.

Solleiro, J., \& Castañón, R. (2005). Competitiveness and innovation systems: the challenges for Mexico's insertion in the global context. Technovation, 25, 1059-1070. 
Tápies, J. (16 de Julio de 2018). IESE. Obtenido de El proceso de sucesión en la empresa familiar: https://www.iese.edu/en/files/recopilacion_sucesion_tcm4-22117.pdf

Valencia-Arias, A., Gutiérrez, L., \& Montoya, L. (2017). Consideraciones acerca del emprendimiento estratégico como síntesis funcional de la identificación de oportunidades y la creación de ventajas competitivas. Revista Virtual Universidad Católica del Norte, 52, 143-159.

Yance, C., Solis, L., Burgos, I., \& Hermida, L. (2017). La importancia de las Pymes en el Ecuador. Revista Observatorio de la Economía Ecuatoriana , 1-17. 